\title{
FTIR Micro-tomography of Five Itokawa Particles and one Primitive Carbonaceous Chondrite
}

Dionnet Z. ${ }^{1}$, Aléon-Toppani A. ${ }^{1}$, Borondics F. ${ }^{2}$, Brunetto R. ${ }^{1}$, Buellet A.C. ${ }^{1}$, Djouadi Z. ${ }^{1}$, King A. ${ }^{3}$ Rubino S. ${ }^{1}$ and Troadec D. ${ }^{4}$

${ }^{1}$ Institut d'Astrophysique Spatiale, CNRS, Université Paris-Saclay, France

${ }^{2}$ SMIS beamline, Synchrotron SOLEIL, France

${ }^{3}$ PSICHE beamline, Synchrotron SOLEIL, France

${ }^{4}$ Institut d'Electronique, de Microélectronique et de Nanotechnologie, Lille, France

Primitive extra-terrestrial materials (asteroidal and cometary particles, meteorites, IDPs) are characterized by a large mineralogical and compositional heterogeneity at different scales (from nm to $\mathrm{mm}$ ) [1], which witnesses the complexity of the pre-accretional (solar nebula) and post-accretional (parent bodies) processes undergone by the small bodies of our Solar System [2]. This heterogeneity has been observed by different techniques such as infrared (IR) micro spectroscopy mapping which is a powerful tool as it is (a) non-destructive, (b) allows comparison with astronomical observations of primitive Solar System small bodies (asteroids, comets) [3] and (c) access both to the mineral and carbonaceous phases. It also allows hyperspectral imaging studies, useful to visualize the spatial distribution of different components, their assembly and thus to deduce constraints about their formation and their evolution in the young Solar System.

Thanks to Focal Plane Array (FPA) detector, 3D hyperspectral micro-tomography, can be performed to access structural information on intact samples [4,5]. So far, this technique has never been applied to Hayabusa-1. Here, we will present the first 3D infrared reconstruction of five particles of Itokawa (RAQD02-0214, RA-QD02-0223, RA-QD02-0232, RA-QD02-0156, and RB-QD04-0046). The FTIR micro analyses are performed at the SMIS beamline of the French Synchrotron SOLEIL using an FPA detector with its Globar internal source. It is complementary to the X-ray micro-tomography previously performed on Hayabusa-1 particles [6]. Novel numerical methods have been developed to deal with a huge quantity of hyperspectral infrared data, we thus are able to obtain the 3D spatial distribution of chemical/mineralogical components (low/high calcium pyroxene, olivine, and plagioclase) and mineralogical properties such as $\mathrm{Fe} / \mathrm{Mg}$ ratio of the olivine.

Another analysis was performed on grains of the Paris meteorite, one of the most primitive carbonaceous chondrite [7]. Using a Focused Ion Beam (FIB), we have developed a new technique of sample preparation to fix them at the extremity of a needle (see Figure 1(a)). This preparation minimized the organic contamination. Then we performed IR 3D hyperspectral micro-tomography to reconstruct the sample at different wavelengths (see Figure 1(b) for a reconstruction representative of the whole matter) and to reconstruct the spatial distribution of the different components. We could then study the spatial correlation between the meteoritic organic and mineral phases at scales down to $\sim 3 \mu \mathrm{m}$. Moreover, X-rays tomography was also performed on the same Paris particles (see Figure 1(c)), at the PSICHE beamline of the synchrotron SOLEIL, to obtain complementary information about the physical properties of the grains (shape, fractures, porosity ...). X-ray analysis were performed at low energy to preserve the organic matter inside our sample. By combining X-ray and FTIR data we could obtain a physico-chemical description of precious grains in a non-destructive way and thus obtain some information about the composition of asteroids. 
Performing FTIR micro-tomography on extraterrestrial samples rich in organic matter, is an important step in view of the sample return of dust particles from carbonaceous asteroids Ryugu by the Hayabusa-2 mission and Bennu by OSIRIS-Rex mission. In the sequence of analyses, micro-FTIR 3D spectral imaging coupled with $\mathrm{X}$-rays tomography can provide a first, powerful non-destructive characterization of whole grains, in order to identify areas of interest and provide useful information before subsequent destructive analyses [8].

\section{References:}

[1] F.J.M. Rietmeijer, Reviews in Mineralogy 36 (1998), (Chapter 2) p. 95

[2] C.M. O’D. Alexander, Geochimica et Cosmochimica Acta 71, Issue 17 (2007), p. 4380-4403.

[3] R. Brunetto et al, Icarus 212 (2011), p. 896-910.

[4] M.J. Nasse et al, Nature method 8 (2011), p. 1585.

[5] M.C. Martin et al, Nature Methods 10 (2013), p. 861-864.

[6] A. Tsuchiyama et al, Science 333 (2011), p. 1125.

[7] R. Hewins et al, Geochimica et Cosmochimica Acta 124 (2014), p. 190-222.

[8] We are grateful to T.Yada, T.Nakamura, C. Sandt and L. Bonal for help and useful discussion. The micro-spectroscopy activities are supported by grants from Région Ile-de-France (DIM-ACAV) and SOLEIL.

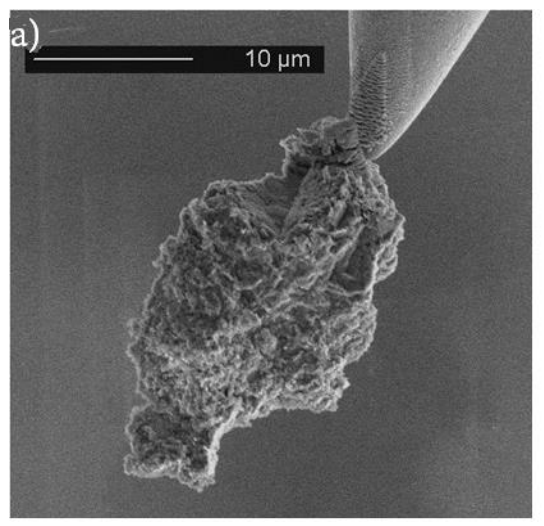

b)

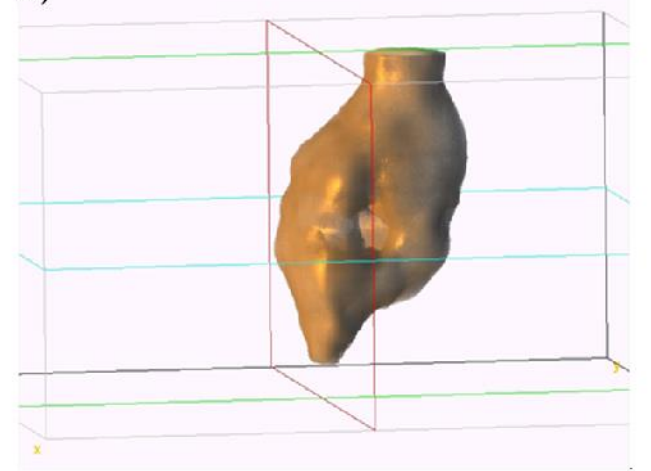

c)

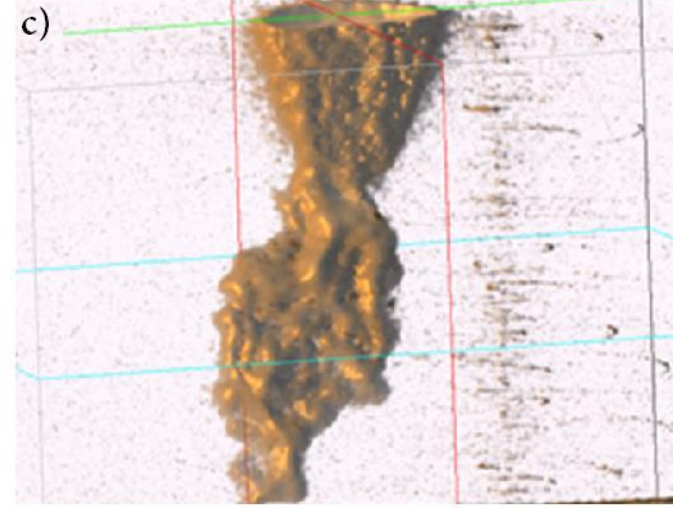

Figure 1. Paris meteorite sample welded at the extremity of the tungsten needle thanks to FIB preparation (a) MET image, (b) IR reconstruction of the continuum at $2.6 \mu \mathrm{m}$ and (c) X-ray reconstruction at $20 \mathrm{keV}$. 